\title{
On Asymptotic Behavior of the Time-Dependent Solution of a Reliability Model
}

\section{Geni Gupur}

\author{
College of Mathematics and Systems Science, Xinjiang University, Urumqi 830046, P.R.China
}

Keywords: The system consisting of two identical units and a repairman, resolvent, eigenvalue

\begin{abstract}
On the basis of our previous work we study asymptotic behavior of the time-dependent solution of a reliability model of two identical units and a repairman and prove the following result: If the repair rate $\mu(\mathrm{x})$ is Lipschitz continuous and there exist two positive constants $\bar{\mu}$ and $\underline{\mu}$ such that $0<\underline{\mu} \leq \mu(x) \leq \bar{\mu}<\infty$, then its time-dependent solution exponentially converges to its steady-state solution.
\end{abstract}

\section{Introduction}

The system consisting of two identical units and a repairman is a common system in operations research. It generalized several basic reliability systems such as a parallel system of two identical units, a cold standby system of two identical units, a warm standby system of two identical units and the $(n-1) / n(G)$ system (see Chapter 4 of Gupur [4]). In 2012, Gupur [4] established a mathematical model to describe the above system by using the supplementary variable technique (see Cao and Cheng [1]), next converted the model into an abstract Cauchy problem by choosing a state space, operators and their domains, thirdly proved that the underlying operator which corresponds to the model generates a positive contraction $\mathrm{C}_{0}$-semigroup which is isometric for the initial value, and therefore deduced that the model has a unique positive time-dependent solution which satisfies the probability condition (its norm is 1 ). Finally, he proved the $\mathrm{C}_{0}$-semigroup is a quasi-compact operator and 0 is an eigenvalue of the underlying operator and its adjoint operator with geometric multiplicity 1. Thus, by using Theorem 2.1 in Nagel [5], p.343, Gupur [4] obtained that the $\mathrm{C}_{0}$-semigroup exponentially converges to a projection. In particular, the time-dependent solution of the model strongly converges to its steady-state solution. So far, no other results have been found in the literature.

In this paper, on the basis of our previous work we firstly study the essential growth bound of the $\mathrm{C}_{0}$-semigroup and show that its essential growth bound is less than a negative number, thus we deduce that 0 is an isolated eigenvalue of the underlying operator and therefore 0 is a pole of its resolvent of order one. Finally, by using the residue theorem we determine the expression of the projection and conclude that the time-dependent solution of the model converges exponentially to its steady-state solution. Idea and method used in this paper are suitable for studying other reliability models which were described finitely many partial differential equations with integral boundary conditions.

According to Gupur [4], p. 143, the system of two identical units and a repairman can be described by the following system of equations:

$$
\frac{d p_{0}(t)}{d t}=-\lambda_{0} p_{0}(t)+\int_{0}^{\infty} p_{1}(x, t) \mu(x) d x,
$$




$$
\begin{aligned}
\frac{\partial p_{1}(x, t)}{\partial t}+\frac{\partial p_{1}(x, t)}{\partial x} & =-\left(\lambda_{1}+\mu(x)\right) p_{1}(x, t) \\
\frac{\partial p_{2}(x, t)}{\partial t}+\frac{\partial p_{2}(x, t)}{\partial x} & =-\mu(x) p_{2}(x, t)+\lambda_{1} p_{1}(x, t) \\
p_{1}(0, t) & =\lambda_{0} p_{0}(t)+\int_{0}^{\infty} p_{2}(x, t) \mu(x) d x \\
p_{2}(0, t) & =0, \\
p_{0}(0) & =\phi_{0} \geq 0, \quad p_{i}(x, 0)=\phi_{i}(x) \geq 0, i=1,2 .
\end{aligned}
$$

Where $(x, t) \in[0, \infty) \times[0, \infty) \cdot \phi_{0}+\sum_{i=1}^{2} \int_{0}^{\infty} \phi_{i}(x) d x=1 . p_{0}(t)$ is the probability that at moment $t$, no units are down. $p_{1}(x, t)$ is the probability that at moment $t$, one unit is down with elapsed repair time $x . p_{2}(x, t) d x$ is the probability that at moment $t$, two units are down and elapsed repair time of the repairing unit lies in $(x, x+d x] . \lambda_{0}$ is the failure rate of a unit and $\lambda_{1}$ is the failure rate of the system. $\mu(x)$ is the repair rate at time $x$ satisfying.

$$
\mu(x) \geq 0, \quad \int_{0}^{\infty} \mu(x) d x=\infty .
$$

We use notations in Gupur [4]. For simplicity, take

$$
\Gamma=\left(\begin{array}{ccc}
e^{-x} & 0 & 0 \\
\lambda_{0} e^{-x} & 0 & \mu(x) \\
0 & 0 & 0
\end{array}\right)
$$

Select a state space as follows:

$$
X=\left\{p \in \mathbb{R} \times L^{1}[0, \infty) \times L^{1}[0, \infty)|\|p\|=| p_{0} \mid+\sum_{i=1}^{2}\left\|p_{i}\right\|_{L^{1}[0, \infty)}\right\} .
$$

It is obvious that $X$ is a Banach space. Moreover, $X$ is a Banach lattice under the following order relation:

$$
p \leq y \Leftrightarrow p_{0} \leq y_{0}, p_{i}(x) \leq y_{i}(x), i=1,2, \quad p, y \in X
$$

In the following we define operators and their domains.

$$
\begin{aligned}
& D(\mathcal{A})=\left\{\begin{array}{l|l}
p \in X & \begin{array}{l}
\frac{d p_{i}(x)}{d x} \in L^{1}[0, \infty), p_{i}(x)(i=1,2) \\
\text { are absolutely continuous } \\
\text { and satisfy } p(0)=\int_{0}^{\infty} \Gamma p(x) d x
\end{array}
\end{array}\right\}, \\
& \mathcal{A}\left(\begin{array}{l}
p_{0} \\
p_{1} \\
p_{2}
\end{array}\right)=\left(\begin{array}{ccc}
-\lambda_{0} & 0 & 0 \\
0 & -\frac{d}{d x}-\left(\lambda_{1}+\mu(x)\right) & 0 \\
0 & 0 & -\frac{d}{d x}-\mu(x)
\end{array}\right)\left(\begin{array}{l}
p_{0} \\
p_{1} \\
p_{2}
\end{array}\right), p \in D(\mathcal{A}) ; \\
& U\left(\begin{array}{l}
p_{0} \\
p_{1} \\
p_{2}
\end{array}\right)=\left(\begin{array}{ccc}
0 & 0 & 0 \\
0 & 0 & 0 \\
0 & \lambda_{1} & 0
\end{array}\right)\left(\begin{array}{l}
p_{0} \\
p_{1} \\
p_{2}
\end{array}\right), \quad D(U)=X ; \\
& E\left(\begin{array}{l}
p_{0} \\
p_{1} \\
p_{2}
\end{array}\right)=\left(\begin{array}{c}
\int_{0}^{\infty} p_{1}(x) \mu(x) d x \\
0 \\
0
\end{array}\right), \quad D(E)=X .
\end{aligned}
$$


Then the above equations (1.1)-(1.6) can be rewritten as an abstract Cauchy problem in $X$ which was given in Gupur [4]:

$$
\left\{\begin{array}{l}
\frac{d p(t)}{d t}=(\mathcal{A}+U+E) p(t), \quad \forall t \in(0, \infty) \\
p(0)=\left(\phi_{0}, \phi_{1}, \phi_{2}\right) .
\end{array}\right.
$$

In 2012, Gupur [4] obtained the following results:

Theorem 1.1. ([4],p.149,158,159) If $M=\sup _{x \in[0, \infty)} \mu(x)<\infty$, then $\mathcal{A}+U$ and $\mathcal{A}+U+E$ generate positive contraction $\mathrm{C}_{0}$-semigroups $\mathrm{S}(\mathrm{t})$ and $\mathrm{T}(\mathrm{t})$ respectively. $\mathrm{T}(\mathrm{t})$ is isometric for ${ }^{p(0) \in D\left(\mathcal{A}^{2}\right)}$, i.e.,

$$
\|T(t) p(0)\|=\|p(0)\|, \quad \forall t \in[0, \infty) .
$$

Hence, the system (1.7) has a unique positive time-dependent solution $p(x, t)=T(t) p(0)$ satisfying

$$
\|p(\cdot, t)\|=1, \quad \forall t \in[0, \infty) .
$$

For $\forall \phi \in X$ define two operators:

$$
\begin{aligned}
& (V(t) \phi)(x)= \begin{cases}0 & x \in[0, t), \\
(S(t) \phi)(x) & x \in[t, \infty),\end{cases} \\
& (W(t) \phi)(x)= \begin{cases}(S(t) \phi)(x) & x \in[0, t), \\
0, & x \in[t, \infty),\end{cases}
\end{aligned}
$$

then $S(t) \phi=V(t) \phi+W(t) \phi, \quad \forall \phi \in X$.

Theorem 1.2. ([4],p.163,172) If $\mu(\mathrm{x})$ is Lipschitz continuous and there exist two positive constants $\bar{\mu}$ and $\underline{\mu}$ such that $0<\underline{\mu} \leq \mu(x) \leq \bar{\mu}<\infty$, then $\mathrm{W}(\mathrm{t})$ is compact on $\mathrm{X}$ and $\mathrm{V}(\mathrm{t})$ satisfies

$$
\|V(t)\| \leq e^{-\min \left\{\lambda_{0}, \underline{\mu}\right\} t} .
$$

Hence, $S(t)$ and $T(t)$ are quasi-compact on $X$. If $\int_{0}^{\infty} \mu(x) e^{-\lambda_{1} x-\int_{0}^{x} \mu(\tau) d \tau} d x<\infty$, then 0 is an eigenvalue of $\mathcal{A}+U+E$ and $(\mathcal{A}+U+E)^{*}$ with geometric multiplicity one. Moreover, $p(x)=$ $\left(p_{0}, p_{1}(x), p_{2}(x)\right)$ is an eigenvector of $\mathcal{A}+U+E$ corresponding to 0 , here

$$
\begin{aligned}
& p_{1}(x)=\frac{\lambda_{0} e^{-\lambda_{1} x-\int_{0}^{x} \mu(\xi) d \xi}}{\int_{0}^{\infty} \mu(x) e^{-\lambda_{1} x-\int_{0}^{x} \mu(\xi) d \xi} d x} p_{0}, \\
& p_{2}(x)=\frac{\lambda_{0} e^{-\int_{0}^{x} \mu(\xi) d \xi}\left(1-e^{-\lambda_{1} x}\right)}{\int_{0}^{\infty} \mu(x) e^{-\lambda_{1} x-\int_{0}^{x} \mu(\xi) d \xi} d x} p_{0} .
\end{aligned}
$$

Theorem 1.3. ([4],p.179) If $\mu(\mathrm{x})$ are Lipschitz continuous and satisfies $0<\underline{\mu} \leq \mu(x) \leq \bar{\mu}<\infty$, then there exist a positive projection $P$ and positive constants $\delta>0, \bar{M} \geq 0$ such that

$$
\|T(t)-\mathbb{P}\| \leq \bar{M} e^{-\delta t},
$$

$$
\text { here } \mathbb{P}=\frac{1}{2 \pi i} \int_{\bar{\Gamma}}(z I-\mathcal{A}-U-E)^{-1} d z, \bar{\Gamma} \text { is a circle with center } 0 \text { and sufficiently small radius. }
$$

In this paper, by discussing the essential growth bound of $\mathrm{T}(\mathrm{t})$ we show that 0 is an isolated eigenvalue of $\mathrm{A}+\mathrm{U}+\mathrm{E}$ and therefore deduce that 0 is a pole of its resolvent of order 1 . Next by considering resolvent of $\mathrm{A}+\mathrm{U}+\mathrm{E}$ and using the residue theorem we obtain the expression of the projection $P$. Finally, we prove that the time-dependent solution of the system (1.7) exponentially converges to its steady-state solution. 


\section{Main Results}

Lemma 2.1. For $\gamma \in \rho(\mathcal{A}+U+E)$ we have

$$
(\gamma I-\mathcal{A}-U-E)^{-1}\left(\begin{array}{c}
z_{0} \\
z_{1} \\
z_{2}
\end{array}\right)=\left(\begin{array}{l}
y_{0} \\
y_{1} \\
y_{2}
\end{array}\right), \quad \forall z \in X
$$

here

$$
\begin{aligned}
& y_{0}=\left\{\int_{0}^{\infty} \mu(x) e^{-\left(\gamma+\lambda_{1}\right) x-\int_{0}^{x} \mu(\xi) d \xi} d x\right. \\
&\left.\times \int_{0}^{\infty} \mu(x) e^{-\gamma x-\int_{0}^{x} \mu(\xi) d \xi} \int_{0}^{x} z_{1}(\eta) e^{\left(\gamma+\lambda_{1}\right) \eta+\int_{0}^{\eta} \mu(\xi) d \xi}\left(e^{-\lambda_{1} \eta}-e^{-\lambda_{1} x}\right) d \eta d x\right\} \\
& /\left\{\gamma\left[1-\int_{0}^{\infty} \mu(x) e^{-\gamma x-\int_{0}^{x} \mu(\xi) d \xi}\left(1-e^{-\lambda_{1} x}\right) d x\right]\right. \\
&\left.+\lambda_{0}\left[1-\int_{0}^{\infty} \mu(x) e^{-\gamma x-\int_{0}^{x} \mu(\xi) d \xi} d x\right]\right\} \\
&+\left\{\int_{0}^{\infty} \mu(x) e^{-\left(\gamma+\lambda_{1}\right) x-\int_{0}^{x} \mu(\xi) d \xi} d x\right. \\
&\left.\times \int_{0}^{\infty} \mu(x) e^{-\gamma x-\int_{0}^{x} \mu(\xi) d \xi} \int_{0}^{x} z_{2}(\tau) e^{\gamma \tau+\int_{0}^{\tau} \mu(\xi) d \xi} d \tau d x\right\} \\
& /\left\{\gamma\left[1-\int_{0}^{\infty} \mu(x) e^{-\gamma x-\int_{0}^{x} \mu(\xi) d \xi}\left(1-e^{-\lambda_{1} x}\right) d x\right]\right. \\
&\left.+\lambda_{0}\left[1-\int_{0}^{\infty} \mu(x) e^{-\gamma x-\int_{0}^{x} \mu(\xi) d \xi} d x\right]\right\} \\
&+\left\{\left[1-\int_{0}^{\infty} \mu(x) e^{-\gamma x-\int_{0}^{x} \mu(\xi) d \xi}\left(1-e^{-\lambda_{1} x}\right) d x\right]\right. \\
&+\left\{\gamma\left[1-\int_{0}^{\infty} \mu(x) e^{-\gamma x-\int_{0}^{x} \mu(\xi) d \xi}\left(1-e^{-\lambda_{1} x}\right) d x\right]\right. \\
&\left.+\lambda_{0}\left[1-\int_{0}^{\infty} \mu(x) e^{-\gamma x-\int_{0}^{x} \mu(\xi) d \xi} d x\right]\right\} \\
&\left.\times \int_{0}^{\infty} \mu(x) e^{-\left(\gamma+\lambda_{1}\right) x-\int_{0}^{x} \mu(\xi) d \xi} \int_{0}^{x} z_{1}(\tau) e^{\left(\gamma+\lambda_{1}\right) \tau+\int_{0}^{\tau} \mu(\xi) d \xi} d \tau d x\right\} \\
& /\left\{\gamma\left[1-\int_{0}^{\infty} \mu(x) e^{-\gamma x-\int_{0}^{x} \mu(\xi) d \xi}\left(1-e^{-\lambda_{1} x}\right) d x\right]\right. \\
&+\int_{0}^{\infty} \mu(x) e^{-\gamma x-\int_{0}^{x} \mu(\xi) d \xi}\left(1-e^{-\lambda_{1} x}\right) d x \\
&-\left(\gamma+\int_{1}\right) x-\int_{0}^{x} \mu(\xi) d \xi \\
&\left.\left.-\gamma(x) e^{-\gamma x-\int_{0}^{x} \mu(\xi) d \xi} d x\right]\right\}
\end{aligned}
$$




$$
\begin{aligned}
& \times \int_{0}^{\infty} \mu(x) e^{-\gamma x-\int_{0}^{x} \mu(\xi) d \xi} \int_{0}^{x} z_{1}(\eta) e^{\left(\gamma+\lambda_{1}\right) \eta+\int_{0}^{\eta} \mu(\xi) d \xi}\left(e^{-\lambda_{1} \eta}-e^{-\lambda_{1} x}\right) d \eta d x \\
& +\frac{e^{-\left(\gamma+\lambda_{1}\right) x-\int_{0}^{x} \mu(\xi) d \xi}}{1-\int_{0}^{\infty} \mu(x) e^{-\gamma x-\int_{0}^{x} \mu(\xi) d \xi}\left(1-e^{-\lambda_{1} x}\right) d x} \\
& \times \int_{0}^{\infty} \mu(x) e^{-\gamma x-\int_{0}^{x} \mu(\xi) d \xi} \int_{0}^{x} z_{2}(\tau) e^{\gamma \tau+\int_{0}^{\tau} \mu(\xi) d \xi} d \tau d x \\
& +e^{-\left(\gamma+\lambda_{1}\right) x-\int_{0}^{x} \mu(\xi) d \xi} \int_{0}^{x} z_{1}(\tau) e^{\left(\gamma+\lambda_{1}\right) \tau+\int_{0}^{\tau} \mu(\xi) d \xi} d \tau \\
y_{2}(x)= & \lambda_{1} e^{-\gamma x-\int_{0}^{x} \mu(\xi) d \xi} \int_{0}^{x} y_{1}(\tau) e^{\gamma \tau+\int_{0}^{\tau} \mu(\xi) d \xi} d \tau \\
& +e^{-\gamma x-\int_{0}^{x} \mu(\xi) d \xi} \int_{0}^{x} z_{2}(\tau) e^{\gamma \tau+\int_{0}^{\tau} \mu(\xi) d \xi} d \tau .
\end{aligned}
$$

Proof. For any given $z \in X$ we consider the equation $(\gamma I-\mathcal{A}-U-E) y=z$ which is equivalent to

$$
\begin{aligned}
& \left(\gamma+\lambda_{0}\right) y_{0}=\int_{0}^{\infty} y_{1}(x) \mu(x) d x+z_{0}, \\
& \frac{d y_{1}(x)}{d x}=-\left(\gamma+\lambda_{1}+\mu(x)\right) y_{1}(x)+z_{1}(x), \\
& \frac{d y_{2}(x)}{d x}=-(\gamma+\mu(x)) y_{2}(x)+\lambda_{1} y_{1}(x)+z_{2}(x), \\
& y_{1}(0)=\lambda_{0} y_{0}+\int_{0}^{\infty} y_{2}(x) \mu(x) d x, \\
& y_{2}(0)=0 .
\end{aligned}
$$

By solving (2.2) and (2.3) we have

$$
\begin{aligned}
& y_{1}(x)=a_{1} e^{-\left(\gamma+\lambda_{1}\right) x-\int_{0}^{x} \mu(\xi) d \xi}+e^{-\left(\gamma+\lambda_{1}\right) x-\int_{0}^{x} \mu(\xi) d \xi} \int_{0}^{x} z_{1}(\tau) e^{\left(\gamma+\lambda_{1}\right) \tau+\int_{0}^{\tau} \mu(\xi) d \xi} d \tau, \\
& y_{2}(x)=a_{2} e^{-\gamma x-\int_{0}^{x} \mu(\xi) d \xi}+e^{-\gamma x-\int_{0}^{x} \mu(\xi) d \xi} \int_{0}^{x}\left[\lambda_{1} y_{1}(\tau)+z_{2}(\tau)\right] e^{\gamma \tau+\int_{0}^{\tau} \mu(\xi) d \xi} d \tau .
\end{aligned}
$$

(2.5) and (2.7) give

$$
\begin{aligned}
y_{2}(x)= & \lambda_{1} e^{-\gamma x-\int_{0}^{x} \mu(\xi) d \xi} \int_{0}^{x} y_{1}(\tau) e^{\gamma \tau+\int_{0}^{\tau} \mu(\xi) d \xi} d \tau \\
& +e^{-\gamma x-\int_{0}^{x} \mu(\xi) d \xi} \int_{0}^{x} z_{2}(\tau) e^{\gamma \tau+\int_{0}^{\tau} \mu(\xi) d \xi} d \tau .
\end{aligned}
$$

By combining (2.8) with (2.4) and (2.6) and using the Fubini theorem we deduce

$$
\begin{aligned}
a_{1}= & \lambda_{0} y_{0}+\lambda_{1} \int_{0}^{\infty} \mu(x) e^{-\gamma x-\int_{0}^{x} \mu(\xi) d \xi} \int_{0}^{x} y_{1}(\tau) e^{\gamma \tau+\int_{0}^{\tau} \mu(\xi) d \xi} d \tau d x \\
& +\int_{0}^{\infty} \mu(x) e^{-\gamma x-\int_{0}^{x} \mu(\xi) d \xi} \int_{0}^{x} z_{2}(\tau) e^{\gamma \tau+\int_{0}^{\tau} \mu(\xi) d \xi} d \tau d x \\
= & \lambda_{0} y_{0}+\lambda_{1} \int_{0}^{\infty} \mu(x) e^{-\gamma x-\int_{0}^{x} \mu(\xi) d \xi} \int_{0}^{x} a_{1} e^{-\lambda_{1} \tau} d \tau d x \\
& +\lambda_{1} \int_{0}^{\infty} \mu(x) e^{-\gamma x-\int_{0}^{x} \mu(\xi) d \xi} \int_{0}^{x} e^{-\lambda_{1} \tau} \int_{0}^{\tau} z_{1}(\eta) e^{\left(\gamma+\lambda_{1}\right) \eta+\int_{0}^{\eta} \mu(\xi) d \xi} d \eta d \tau d x
\end{aligned}
$$




$$
\begin{aligned}
& +\int_{0}^{\infty} \mu(x) e^{-\gamma x-\int_{0}^{x} \mu(\xi) d \xi} \int_{0}^{x} z_{2}(\tau) e^{\gamma \tau+\int_{0}^{\tau} \mu(\xi) d \xi} d \tau d x \\
& =\lambda_{0} y_{0}+a_{1} \int_{0}^{\infty} \mu(x) e^{-\gamma x-\int_{0}^{x} \mu(\xi) d \xi}\left(1-e^{-\lambda_{1} x}\right) d x \\
& +\lambda_{1} \int_{0}^{\infty} \mu(x) e^{-\gamma x-\int_{0}^{x} \mu(\xi) d \xi} \int_{0}^{x} z_{1}(\eta) e^{\left(\gamma+\lambda_{1}\right) \eta+\int_{0}^{\eta} \mu(\xi) d \xi} \int_{\eta}^{x} e^{-\lambda_{1} \tau} d \tau d \eta d x \\
& +\int_{0}^{\infty} \mu(x) e^{-\gamma x-\int_{0}^{x} \mu(\xi) d \xi} \int_{0}^{x} z_{2}(\tau) e^{\gamma \tau+\int_{0}^{\tau} \mu(\xi) d \xi} d \tau d x \\
& =\lambda_{0} y_{0}+a_{1} \int_{0}^{\infty} \mu(x) e^{-\gamma x-\int_{0}^{x} \mu(\xi) d \xi}\left(1-e^{-\lambda_{1} x}\right) d x \\
& +\int_{0}^{\infty} \mu(x) e^{-\gamma x-\int_{0}^{x} \mu(\xi) d \xi} \int_{0}^{x} z_{1}(\eta) e^{\left(\gamma+\lambda_{1}\right) \eta+\int_{0}^{\eta} \mu(\xi) d \xi}\left(e^{-\lambda_{1} \eta}-e^{-\lambda_{1} x}\right) d \eta d x \\
& +\int_{0}^{\infty} \mu(x) e^{-\gamma x-\int_{0}^{x} \mu(\xi) d \xi} \int_{0}^{x} z_{2}(\tau) e^{\gamma \tau+\int_{0}^{\tau} \mu(\xi) d \xi} d \tau d x \\
& { }_{1}=\frac{\lambda_{0}}{1-\int_{0}^{\infty} \mu(x) e^{-\gamma x-\int_{0}^{x} \mu(\xi) d \xi}\left(1-e^{-\lambda_{1} x}\right) d x} y_{0} \\
& +\frac{1}{1-\int_{0}^{\infty} \mu(x) e^{-\gamma x-\int_{0}^{x} \mu(\xi) d \xi}\left(1-e^{-\lambda_{1} x}\right) d x} \\
& \times \int_{0}^{\infty} \mu(x) e^{-\gamma x-\int_{0}^{x} \mu(\xi) d \xi} \int_{0}^{x} z_{1}(\eta) e^{\left(\gamma+\lambda_{1}\right) \eta+\int_{0}^{\eta} \mu(\xi) d \xi}\left(e^{-\lambda_{1} \eta}-e^{-\lambda_{1} x}\right) d \eta d x \\
& +\frac{1}{1-\int_{0}^{\infty} \mu(x) e^{-\gamma x-\int_{0}^{x} \mu(\xi) d \xi}\left(1-e^{-\lambda_{1} x}\right) d x} \\
& \times \int_{0}^{\infty} \mu(x) e^{-\gamma x-\int_{0}^{x} \mu(\xi) d \xi} \int_{0}^{x} z_{2}(\tau) e^{\gamma \tau+\int_{0}^{\tau} \mu(\xi) d \xi} d \tau d x .
\end{aligned}
$$

By inserting (2.9) into (2.6) we derive

$$
\begin{aligned}
y_{1}(x)= & \frac{\lambda_{0} e^{-\left(\gamma+\lambda_{1}\right) x-\int_{0}^{x} \mu(\xi) d \xi}}{1-\int_{0}^{\infty} \mu(x) e^{-\gamma x-\int_{0}^{x} \mu(\xi) d \xi}\left(1-e^{-\lambda_{1} x}\right) d x} y_{0} \\
& +\frac{e^{-\left(\gamma+\lambda_{1}\right) x-\int_{0}^{x} \mu(\xi) d \xi}}{1-\int_{0}^{\infty} \mu(x) e^{-\gamma x-\int_{0}^{x} \mu(\xi) d \xi}\left(1-e^{-\lambda_{1} x}\right) d x} \\
& \times \int_{0}^{\infty} \mu(x) e^{-\gamma x-\int_{0}^{x} \mu(\xi) d \xi} \int_{0}^{x} z_{1}(\eta) e^{\left(\gamma+\lambda_{1}\right) \eta+\int_{0}^{\eta} \mu(\xi) d \xi}\left(e^{-\lambda_{1} \eta}-e^{-\lambda_{1} x}\right) d \eta d x \\
& +\frac{e^{-\left(\gamma+\lambda_{1}\right) x-\int_{0}^{x} \mu(\xi) d \xi}}{1-\int_{0}^{\infty} \mu(x) e^{-\gamma x-\int_{0}^{x} \mu(\xi) d \xi}\left(1-e^{-\lambda_{1} x}\right) d x} \\
& \times \int_{0}^{\infty} \mu(x) e^{-\gamma x-\int_{0}^{x} \mu(\xi) d \xi} \int_{0}^{x} z_{2}(\tau) e^{\gamma \tau+\int_{0}^{\tau} \mu(\xi) d \xi} d \tau d x \\
& +e^{-\left(\gamma+\lambda_{1}\right) x-\int_{0}^{x} \mu(\xi) d \xi} \int_{0}^{x} z_{1}(\tau) e^{\left(\gamma+\lambda_{1}\right) \tau+\int_{0}^{\tau} \mu(\xi) d \xi} d \tau .
\end{aligned}
$$

By substituting (2.10) into (2.1) we determine 
International Frontier Science Letters Vol. 2

7

$$
\begin{aligned}
& \left(\gamma+\lambda_{0}\right) y_{0}=\frac{\int_{0}^{\infty} \mu(x) e^{-\left(\gamma+\lambda_{1}\right) x-\int_{0}^{x} \mu(\xi) d \xi} d x}{1-\int_{0}^{\infty} \mu(x) e^{-\gamma x-\int_{0}^{x} \mu(\xi) d \xi}\left(1-e^{-\lambda_{1} x}\right) d x} \lambda_{0} y_{0} \\
& +\frac{\int_{0}^{\infty} \mu(x) e^{-\left(\gamma+\lambda_{1}\right) x-\int_{0}^{x} \mu(\xi) d \xi} d x}{1-\int_{0}^{\infty} \mu(x) e^{-\gamma x-\int_{0}^{x} \mu(\xi) d \xi}\left(1-e^{-\lambda_{1} x}\right) d x} \\
& \times \int_{0}^{\infty} \mu(x) e^{-\gamma x-\int_{0}^{x} \mu(\xi) d \xi} \int_{0}^{x} z_{1}(\eta) e^{\left(\gamma+\lambda_{1}\right) \eta+\int_{0}^{\eta} \mu(\xi) d \xi}\left(e^{-\lambda_{1} \eta}-e^{-\lambda_{1} x}\right) d \eta d x \\
& +\frac{\int_{0}^{\infty} \mu(x) e^{-\left(\gamma+\lambda_{1}\right) x-\int_{0}^{x} \mu(\xi) d \xi} d x}{1-\int_{0}^{\infty} \mu(x) e^{-\gamma x-\int_{0}^{x} \mu(\xi) d \xi}\left(1-e^{-\lambda_{1} x}\right) d x} \\
& \times \int_{0}^{\infty} \mu(x) e^{-\gamma x-\int_{0}^{x} \mu(\xi) d \xi} \int_{0}^{x} z_{2}(\tau) e^{\gamma \tau+\int_{0}^{\tau} \mu(\xi) d \xi} d \tau d x \\
& +\int_{0}^{\infty} \mu(x) e^{-\left(\gamma+\lambda_{1}\right) x-\int_{0}^{x} \mu(\xi) d \xi} \int_{0}^{x} z_{1}(\tau) e^{\left(\gamma+\lambda_{1}\right) \tau+\int_{0}^{\tau} \mu(\xi) d \xi} d \tau d x \\
& +z_{0} \\
& \Longrightarrow\left(\gamma+\lambda_{0}-\frac{\lambda_{0} \int_{0}^{\infty} \mu(x) e^{-\left(\gamma+\lambda_{1}\right) x-\int_{0}^{x} \mu(\xi) d \xi} d x}{1-\int_{0}^{\infty} \mu(x) e^{-\gamma x-\int_{0}^{x} \mu(\xi) d \xi}\left(1-e^{-\lambda_{1} x}\right) d x}\right) y_{0} \\
& =\frac{\int_{0}^{\infty} \mu(x) e^{-\left(\gamma+\lambda_{1}\right) x-\int_{0}^{x} \mu(\xi) d \xi} d x}{1-\int_{0}^{\infty} \mu(x) e^{-\gamma x-\int_{0}^{x} \mu(\xi) d \xi}\left(1-e^{-\lambda_{1} x}\right) d x} \\
& \times \int_{0}^{\infty} \mu(x) e^{-\gamma x-\int_{0}^{x} \mu(\xi) d \xi} \int_{0}^{x} z_{1}(\eta) e^{\left(\gamma+\lambda_{1}\right) \eta+\int_{0}^{\eta} \mu(\xi) d \xi}\left(e^{-\lambda_{1} \eta}-e^{-\lambda_{1} x}\right) d \eta d x \\
& +\frac{\int_{0}^{\infty} \mu(x) e^{-\left(\gamma+\lambda_{1}\right) x-\int_{0}^{x} \mu(\xi) d \xi} d x}{1-\int_{0}^{\infty} \mu(x) e^{-\gamma x-\int_{0}^{x} \mu(\xi) d \xi}\left(1-e^{-\lambda_{1} x}\right) d x} \\
& \times \int_{0}^{\infty} \mu(x) e^{-\gamma x-\int_{0}^{x} \mu(\xi) d \xi} \int_{0}^{x} z_{2}(\tau) e^{\gamma \tau+\int_{0}^{\tau} \mu(\xi) d \xi} d \tau d x \\
& +\int_{0}^{\infty} \mu(x) e^{-\left(\gamma+\lambda_{1}\right) x-\int_{0}^{x} \mu(\xi) d \xi} \int_{0}^{x} z_{1}(\tau) e^{\left(\gamma+\lambda_{1}\right) \tau+\int_{0}^{\tau} \mu(\xi) d \xi} d \tau d x \\
& +z_{0} \\
& \Longrightarrow \\
& y_{0}=\left\{\int_{0}^{\infty} \mu(x) e^{-\left(\gamma+\lambda_{1}\right) x-\int_{0}^{x} \mu(\xi) d \xi} d x\right. \\
& \left.\times \int_{0}^{\infty} \mu(x) e^{-\gamma x-\int_{0}^{x} \mu(\xi) d \xi} \int_{0}^{x} z_{1}(\eta) e^{\left(\gamma+\lambda_{1}\right) \eta+\int_{0}^{\eta} \mu(\xi) d \xi}\left(e^{-\lambda_{1} \eta}-e^{-\lambda_{1} x}\right) d \eta d x\right\} \\
& /\left\{\gamma\left[1-\int_{0}^{\infty} \mu(x) e^{-\gamma x-\int_{0}^{x} \mu(\xi) d \xi}\left(1-e^{-\lambda_{1} x}\right) d x\right]\right. \\
& \left.+\lambda_{0}\left[1-\int_{0}^{\infty} \mu(x) e^{-\gamma x-\int_{0}^{x} \mu(\xi) d \xi} d x\right]\right\} \\
& +\left\{\int_{0}^{\infty} \mu(x) e^{-\left(\gamma+\lambda_{1}\right) x-\int_{0}^{x} \mu(\xi) d \xi} d x\right. \\
& \left.\times \int_{0}^{\infty} \mu(x) e^{-\gamma x-\int_{0}^{x} \mu(\xi) d \xi} \int_{0}^{x} z_{2}(\tau) e^{\gamma \tau+\int_{0}^{\tau} \mu(\xi) d \xi} d \tau d x\right\} \\
& /\left\{\gamma\left[1-\int_{0}^{\infty} \mu(x) e^{-\gamma x-\int_{0}^{x} \mu(\xi) d \xi}\left(1-e^{-\lambda_{1} x}\right) d x\right]\right. \\
& \left.+\lambda_{0}\left[1-\int_{0}^{\infty} \mu(x) e^{-\gamma x-\int_{0}^{x} \mu(\xi) d \xi} d x\right]\right\}
\end{aligned}
$$




$$
\begin{aligned}
& +\left\{\left[1-\int_{0}^{\infty} \mu(x) e^{-\gamma x-\int_{0}^{x} \mu(\xi) d \xi}\left(1-e^{-\lambda_{1} x}\right) d x\right]\right. \\
& \left.\times \int_{0}^{\infty} \mu(x) e^{-\left(\gamma+\lambda_{1}\right) x-\int_{0}^{x} \mu(\xi) d \xi} \int_{0}^{x} z_{1}(\tau) e^{\left(\gamma+\lambda_{1}\right) \tau+\int_{0}^{\tau} \mu(\xi) d \xi} d \tau d x\right\} \\
& /\left\{\gamma\left[1-\int_{0}^{\infty} \mu(x) e^{-\gamma x-\int_{0}^{x} \mu(\xi) d \xi}\left(1-e^{-\lambda_{1} x}\right) d x\right]\right. \\
& \left.+\lambda_{0}\left[1-\int_{0}^{\infty} \mu(x) e^{-\gamma x-\int_{0}^{x} \mu(\xi) d \xi} d x\right]\right\} \\
& +\left\{\left[1-\int_{0}^{\infty} \mu(x) e^{-\gamma x-\int_{0}^{x} \mu(\xi) d \xi}\left(1-e^{-\lambda_{1} x}\right)\right] z_{0}\right\} \\
& /\left\{\gamma\left[1-\int_{0}^{\infty} \mu(x) e^{-\gamma x-\int_{0}^{x} \mu(\xi) d \xi}\left(1-e^{-\lambda_{1} x}\right) d x\right]\right. \\
& \left.+\lambda_{0}\left[1-\int_{0}^{\infty} \mu(x) e^{-\gamma x-\int_{0}^{x} \mu(\xi) d \xi} d x\right]\right\} .
\end{aligned}
$$

(2.11), (2.10) and (2.8) are just the result of this lemma.

Theorem 2.2. If $\mu(\mathrm{x})$ is Lipschitz continuous and there exist two positive constants $\bar{\mu}$ and $\underline{\mu}$ such that $0<\mu \leq \mu(x) \leq \bar{\mu}<\infty$, then the time-dependent solution of the system (1.7) exponentially converges to its steady-state solution, i.e.,

$$
\|p(\cdot, t)-p(\cdot)\| \leq \bar{M} e^{-\delta t}, \quad \forall t \geq 0
$$

Proof. Theorem 1.2 implies

$$
\begin{aligned}
\|S(t)-W(t)\| & =\|V(t)\| \leq e^{-\min \left\{\lambda_{0}, \underline{\mu}\right\} t} \Rightarrow \ln \|S(t)-W(t)\|=\ln \|V(t)\| \\
& \leq-\min \left\{\lambda_{0}, \underline{\mu}\right\} t \Rightarrow \frac{\ln \|S(t)-W(t)\|}{t} \leq-\min \left\{\lambda_{0}, \underline{\mu}\right\} .
\end{aligned}
$$

From which together with Proposition 2.10 in Engel and Nagel [2], p. 258 we know that $\omega_{\text {ess }}(S(t))\left(\right.$ i.e., $\left.\omega_{\text {ess }}(A+U)\right)$, the essential growth bound of $S(t)(i . e ., A+U)$, satisfies

$$
\omega_{e s s}(S(t)) \leq-\min \left\{\lambda_{0}, \underline{\mu}\right\} .
$$

This together with Corollary 2.11 in [2], p. 258, Theorem 1.2 and $0 \in \sigma(\mathcal{A}+U+E) \cap\{\gamma \in \mathbb{C} \mid \operatorname{Re} \gamma>$ $\left.\omega_{\text {ess }}(\mathcal{A}+U+E)\right\}$ imply that 0 is an isolated eigenvalue of $\mathcal{A}+U+E$ with algebraic multiplicity one, that is to say, 0 is a pole of $(\gamma I-\mathcal{A}-U-E)^{-1}$ of order 1 . Hence, Theorem 1.3 and the residue thporem give, for the initial value $p(0)=\phi \in D\left(\mathcal{A}^{2}\right)$,

$$
\mathbb{P} \phi(x)=\lim _{\gamma \rightarrow 0} \gamma(\gamma I-\mathcal{A}-U-E)^{-1} \phi(x) .
$$

In the following we determine the above limit. By using the l'Hospital rule and

$$
\int_{0}^{\infty} \mu(x) e^{-\int_{0}^{x} \mu(\xi) d \xi} d x=-\left.e^{-\int_{0}^{x} \mu(\xi) d \xi}\right|_{0} ^{\infty}=1
$$


we calculate

$$
\begin{aligned}
\lim _{\gamma \rightarrow 0} \gamma /\left\{\gamma\left[1-\int_{0}^{\infty} \mu(x) e^{-\gamma x-\int_{0}^{x} \mu(\xi) d \xi}\left(1-e^{-\lambda_{1} x}\right) d x\right]\right. \\
\left.\quad+\lambda_{0}\left[1-\int_{0}^{\infty} \mu(x) e^{-\gamma x-\int_{0}^{x} \mu(\xi) d \xi} d x\right]\right\} \\
=\lim _{\gamma \rightarrow 0} 1 /\left\{1-\int_{0}^{\infty} \mu(x) e^{-\gamma x-\int_{0}^{x} \mu(\xi) d \xi}\left(1-e^{-\lambda_{1} x}\right) d x\right. \\
\quad+\gamma \int_{0}^{\infty} x \mu(x) e^{-\gamma x-\int_{0}^{x} \mu(\xi) d \xi}\left(1-e^{-\lambda_{1} x}\right) d x \\
\left.\quad+\lambda_{0} \int_{0}^{\infty} x \mu(x) e^{-\gamma x-\int_{0}^{x} \mu(\xi) d \xi} d x\right\} \\
=\frac{1-\int_{0}^{\infty} \mu(x) e^{-\int_{0}^{x} \mu(\xi) d \xi}\left(1-e^{-\lambda_{1} x}\right) d x+\lambda_{0} \int_{0}^{\infty} x \mu(x) e^{-\int_{0}^{x} \mu(\xi) d \xi} d x}{1} \\
=\frac{\int_{0}^{\infty} \mu(x) e^{-\lambda_{1} x-\int_{0}^{x} \mu(\xi) d \xi} d x+\lambda_{0} \int_{0}^{\infty} x \mu(x) e^{-\int_{0}^{x} \mu(\xi) d \xi} d x}{}
\end{aligned}
$$

By (2.14), lemma 2.1, the Fubini theorem and

$$
\begin{aligned}
& \int_{0}^{\infty} \mu(x) e^{-\int_{0}^{x} \mu(\xi) d \xi} d x=1, \phi_{0}+\sum_{i=1}^{2} \int_{0}^{\infty} \phi_{i}(x) d x=1, \\
& \int_{0}^{\infty} \mu(x) e^{-\int_{0}^{x} \mu(\xi) d \xi} \int_{0}^{x} \phi_{i}(\tau) e^{\int_{0}^{\tau} \mu(\xi) d \xi} d \tau d x \\
& =\int_{0}^{\infty} \phi_{i}(\tau) e^{\int_{0}^{\tau} \mu(\xi) d \xi} \int_{\tau}^{\infty} \mu(x) e^{-\int_{0}^{x} \mu(\xi) d \xi} d x d \tau \\
& =\int_{0}^{\infty} \phi_{i}(\tau) e^{\int_{0}^{\tau} \mu(\xi) d \xi}\left(-\left.e^{-\int_{0}^{x} \mu(\xi) d \xi}\right|_{x=\tau} ^{x=\infty}\right) d \tau \\
& =\int_{0}^{\infty} \phi_{i}(\tau) d \tau=\int_{0}^{\infty} \phi_{i}(x) d x, \quad i=1,2
\end{aligned}
$$

we derive

$$
\begin{aligned}
\lim _{\gamma \rightarrow 0} \gamma y_{0}= & \left\{\int_{0}^{\infty} \mu(x) e^{-\lambda_{1} x-\int_{0}^{x} \mu(\xi) d \xi} d x\right. \\
& \left.\times \int_{0}^{\infty} \mu(x) e^{-\int_{0}^{x} \mu(\xi) d \xi} \int_{0}^{x} \phi_{1}(\eta) e^{\lambda_{1} \eta+\int_{0}^{\eta} \mu(\xi) d \xi}\left(e^{-\lambda_{1} \eta}-e^{-\lambda_{1} x}\right) d \eta d x\right\} \\
& /\left\{\int_{0}^{\infty} \mu(x) e^{-\lambda_{1} x-\int_{0}^{x} \mu(\xi) d \xi} d x+\lambda_{0} \int_{0}^{\infty} x \mu(x) e^{-\int_{0}^{x} \mu(\xi) d \xi} d x\right\} \\
& +\left\{\int_{0}^{\infty} \mu(x) e^{-\lambda_{1} x-\int_{0}^{x} \mu(\xi) d \xi} d x\right. \\
& \left.\times \int_{0}^{\infty} \mu(x) e^{-\int_{0}^{x} \mu(\xi) d \xi} \int_{0}^{x} \phi_{2}(\tau) e^{\int_{0}^{\tau} \mu(\xi) d \xi} d \tau d x\right\} \\
& /\left\{\int_{0}^{\infty} \mu(x) e^{-\lambda_{1} x-\int_{0}^{x} \mu(\xi) d \xi} d x+\lambda_{0} \int_{0}^{\infty} x \mu(x) e^{-\int_{0}^{x} \mu(\xi) d \xi} d x\right\} \\
& +\left\{\left[1-\int_{0}^{\infty} \mu(x) e^{-\int_{0}^{x} \mu(\xi) d \xi}\left(1-e^{-\lambda_{1} x}\right) d x\right]\right.
\end{aligned}
$$




$$
\begin{aligned}
& \left.\times \int_{0}^{\infty} \mu(x) e^{-\lambda_{1} x-\int_{0}^{x} \mu(\xi) d \xi} \int_{0}^{x} \phi_{1}(\tau) e^{\lambda_{1} \tau+\int_{0}^{\tau} \mu(\xi) d \xi} d \tau d x\right\} \\
& /\left\{\int_{0}^{\infty} \mu(x) e^{-\lambda_{1} x-\int_{0}^{x} \mu(\xi) d \xi} d x+\lambda_{0} \int_{0}^{\infty} x \mu(x) e^{-\int_{0}^{x} \mu(\xi) d \xi} d x\right\} \\
& +\left\{\left[1-\int_{0}^{\infty} \mu(x) e^{-\int_{0}^{x} \mu(\xi) d \xi} d x\left(1-e^{-\lambda_{1} x}\right) d x\right] \phi_{0}\right\} \\
& /\left\{\int_{0}^{\infty} \mu(x) e^{-\lambda_{1} x-\int_{0}^{x} \mu(\xi) d \xi} d x+\lambda_{0} \int_{0}^{\infty} x \mu(x) e^{-\int_{0}^{x} \mu(\xi) d \xi} d x\right\} \\
& =\left\{\int_{0}^{\infty} \mu(x) e^{-\lambda_{1} x-\int_{0}^{x} \mu(\xi) d \xi} d x \int_{0}^{\infty} \mu(x) e^{-\int_{0}^{x} \mu(\xi) d \xi} \int_{0}^{x} \phi_{1}(\tau) e^{\int_{0}^{\tau} \mu(\xi) d \xi} d \tau d x\right. \\
& -\int_{0}^{\infty} \mu(x) e^{-\lambda_{1} x-\int_{0}^{x} \mu(\xi) d \xi} d x \int_{0}^{\infty} \mu(x) e^{-\lambda_{1} x-\int_{0}^{x} \mu(\xi) d \xi} \int_{0}^{x} \phi_{1}(\tau) e^{\lambda_{1} \tau+\int_{0}^{\tau} \mu(\xi) d \xi} d \tau d x \\
& +\int_{0}^{\infty} \mu(x) e^{-\lambda_{1} x-\int_{0}^{x} \mu(\xi) d \xi} d x \int_{0}^{\infty} \phi_{2}(x) d x \\
& +\int_{0}^{\infty} \mu(x) e^{-\lambda_{1} x-\int_{0}^{x} \mu(\xi) d \xi} d x \int_{0}^{\infty} \mu(x) e^{-\lambda_{1} x-\int_{0}^{x} \mu(\xi) d \xi} \int_{0}^{x} \phi_{1}(\tau) e^{\lambda \tau+\int_{0}^{\tau} \mu(\xi) d \xi} d \tau d x \\
& \left.+\phi_{0} \int_{0}^{\infty} \mu(x) e^{-\lambda_{1} x-\int_{0}^{x} \mu(\xi) d \xi} d x\right\} \\
& /\left\{\int_{0}^{\infty} \mu(x) e^{-\lambda_{1} x-\int_{0}^{x} \mu(\xi) d \xi} d x+\lambda_{0} \int_{0}^{\infty} x \mu(x) e^{-\int_{0}^{x} \mu(\xi) d \xi} d x\right\} \\
& =\left[\phi_{0}+\int_{0}^{\infty} \phi_{1}(x) d x+\int_{0}^{\infty} \phi_{2}(x) d x\right] \\
& \times \frac{\int_{0}^{\infty} \mu(x) e^{-\lambda_{1} x-\int_{0}^{x} \mu(\xi) d \xi} d x}{\int_{0}^{\infty} \mu(x) e^{-\lambda_{1} x-\int_{0}^{x} \mu(\xi) d \xi} d x+\lambda_{0} \int_{0}^{\infty} x \mu(x) e^{-\int_{0}^{x} \mu(\xi) d \xi} d x} \\
& =\frac{\int_{0}^{\infty} \mu(x) e^{-\lambda_{1} x-\int_{0}^{x} \mu(\xi) d \xi} d x}{\int_{0}^{\infty} \mu(x) e^{-\lambda_{1} x-\int_{0}^{x} \mu(\xi) d \xi} d x+\lambda_{0} \int_{0}^{\infty} x \mu(x) e^{-\int_{0}^{x} \mu(\xi) d \xi} d x} \\
& :=p_{0} \text {. }
\end{aligned}
$$

This together with Lemma 2.1, (2.15) and (2.16) give, noting that we have taken $p_{0}$ in Theorem 1.2 as (2.17),

$$
\begin{aligned}
\lim _{\gamma \rightarrow 0} \gamma y_{1}(x)= & \frac{\lambda_{0} e^{-\lambda_{1} x-\int_{0}^{x} \mu(\xi) d \xi}}{1-\int_{0}^{\infty} \mu(x) e^{-\int_{0}^{x} \mu(\xi) d \xi}\left(1-e^{-\lambda_{1} x}\right) d x} \\
& \times \frac{\int_{0}^{\infty} \mu(x) e^{-\lambda_{1} x-\int_{0}^{x} \mu(\xi) d \xi} d x}{\int_{0}^{\infty} \mu(x) e^{-\lambda_{1} x-\int_{0}^{x} \mu(\xi) d \xi} d x+\lambda_{0} \int_{0}^{\infty} x \mu(x) e^{-\int_{0}^{x} \mu(\xi) d \xi} d x} \\
= & \frac{\lambda_{0} e^{-\lambda_{1} x-\int_{0}^{x} \mu(\xi) d \xi}}{\int_{0}^{\infty} \mu(x) e^{-\lambda_{1} x-\int_{0}^{x} \mu(\xi) d \xi} d x+\lambda_{0} \int_{0}^{\infty} x \mu(x) e^{-\int_{0}^{x} \mu(\xi) d \xi} d x} \\
= & p_{1}(x) .
\end{aligned}
$$

From this together with Lemma 2.1, (2.15) and (2.16) we derive, noting that we have taken $p_{0}$ in Theorem 1.2 as (2.17),

$$
\lim _{\gamma \rightarrow 0} \gamma y_{2}(x)=\frac{\lambda_{1} e^{-\int_{0}^{x} \mu(\xi) d \xi} \int_{0}^{x} \lambda_{0} e^{-\lambda_{1} \tau} d \tau}{\int_{0}^{\infty} \mu(x) e^{-\lambda_{1} x-\int_{0}^{x} \mu(\xi) d \xi} d x+\lambda_{0} \int_{0}^{\infty} x \mu(x) e^{-\int_{0}^{x} \mu(\xi) d \xi} d x}
$$




$$
\begin{aligned}
& =\frac{\lambda_{0} e^{-\int_{0}^{x} \mu(\xi) d \xi}\left(1-e^{-\lambda_{1} x}\right)}{\int_{0}^{\infty} \mu(x) e^{-\lambda_{1} x-\int_{0}^{x} \mu(\xi) d \xi} d x+\lambda_{0} \int_{0}^{\infty} x \mu(x) e^{-\int_{0}^{x} \mu(\xi) d \xi} d x} \\
& =p_{2}(x) .
\end{aligned}
$$

By combining Theorem 1.2 with (2.17) (2.19) and (2.13) we obtain

$$
\mathbb{P} \phi(x)=p(x) \text {. }
$$

From Theorem 1.1, (2.20), Theorem 1.3 and $\phi_{0}+\sum_{i=1}^{2} \int_{0}^{\infty} \phi_{i}(x) d x=1$ we conclude

$$
\begin{aligned}
\|p(\cdot, t)-p(\cdot)\| & =\|T(t) \phi(\cdot)-\mathbb{P} \phi(\cdot)\| \leq\|T(t)-\mathbb{P}\|\|\phi\| \\
& \leq \bar{M} e^{-\delta t}\|\phi\|=\bar{M} e^{-\delta t}, \quad \forall t \geq 0 .
\end{aligned}
$$

\section{Discussion}

In Gupur [3], p. 133, by discussing spectra of the operator on the left complex plane which corresponds to the system consisting of a reliable machine, an unreliable machine and a storage buffer with finite capacity when the failure rate is a constant, we deduced that its time-dependent solution exponentially converges its steady-state solution. Our idea and method of this paper are suitable to study this model.

It is easy to see that if $\underline{\mu}=\inf _{x \in[0, \infty)} \mu(x)=0$, then Theorem 1.2, Theorem 1.3 and Theorem 2.2 do not hold. So, when $\underline{\mu}=\inf _{x \in[0, \infty)} \mu(x)=0$ it is worth studying asymptotic behavior of the time-dependent solution of the system (1.7).

\section{Acknowledgements}

This work was supported by the National Natural Science Foundation of China (No: 11371303 ).

\section{References}

[1] J. H. Cao and K. Cheng. Introduction to Reliability Mathematics. Higher Education Press, Beijing, 2006.

[2] K. J. Engel and R. Nagel. One-Parameter Semigroups for Linear Evolution Equations. Springer, New York, 2000.

[3] G. Gupur. Functional Analysis Methods for Reliability Models. Springer, Basel, 2011.

[4] G. Gupur. Mathematical Methods in Reliability Theory. Xinjiang University Press, Urumqi, 2012.

[5] R. Nagel. One-Parameter Semigroups of Positive Operators. Springer, Berlin, 1986. 